\title{
Efecto de cocción en horno convencional y microondas sobre rancidez, pérdida por cocción y diferencia sensorial de hamburguesas
}

\section{Effect of conventional oven versus microwave cooking on rancidity, cooking loss and sensory difference of hamburger patties}

\begin{abstract}
RESUMEN
El uso de microondas está muy extendido a nivel doméstico gracias a su rapidez y facilidad de uso. El objetivo del presente trabajo es comparar el efecto de cocción de hamburguesas de distintos tipos de carne, en microondas y en horno convencional, sobre la pérdida de peso y estabilidad oxidativa. Además, determinar si existen diferencias desde el punto de vista sensorial aplicando una prueba triangular, comparando 2 productos presentados en tríos, uno que corresponde a hamburguesa cocida en microondas y otro a hamburguesa cocida en horno convencional. Se adquirieron hamburguesas de pollo, pavo, cerdo y vacuno en el comercio, se cocinaron en horno convencional y en microondas. Se calculó pérdida por cocción, se evaluó la rancidez y diferencias sensoriales entre hamburguesas del mismo tipo de carne, cocidas en distinto tipo de horno. La pérdida por cocción fue menor en hamburguesas cocidas en microondas. La acidez e índice de peróxido aumentaron en las muestras cocidas en ambos tipos de hornos respecto a hamburguesas crudas. La acidez fue mayor en hamburguesas cocidas en horno convencional, y el índice de peróxido fue mayor en hamburguesas cocidas en microondas, llegando a 10,6 meq $\mathrm{O}_{2} / \mathrm{kg}$. En el análisis sensorial se encontraron diferencias significativas $(p<0,05)$ entre las hamburguesas cocidas en horno convencional y en microondas. Resulta mejor opción cocinar hamburguesas en horno convencional, ya que el índice de peróxidos es menor que al cocinar en microondas; dentro del tipo de hamburguesas, son más estables las de vacuno y cerdo frente a la oxidación.

Palabras clave: Índice de peróxido; Hamburguesas; Microondas; Rancidez; Sensorial.
\end{abstract}

\footnotetext{
ABSTRACT

The use of microwaves is very widespread at the domestic level thanks to its speed and ease of use. The aim of this study was to compare cooking loss, rancidity and sensory differences for hamburger patties cooked in conventional versus microwave ovens. We also determined sensorial differences with the triangular test, comparing two products presented in threesomes, one that corresponded to a patty cooked in the microwave and the other in a conventional
}

Vilma Quitral1*, María Luisa Donoso ${ }^{2}$, Paula Jiménez ${ }^{3}$.

1. Escuela de Nutrición y Dietética. Facultad de Salud. Universidad Santo Tomás. Santiago, Chile.

2. Departamento de Desarrollo. Alimentos Doubleland S.A. Santiago, Chile.

3. Departamento de Nutrición. Facultad de Medicina. Universidad de Chile. Santiago, Chile.

*Dirigir correspondencia a: Vilma Quitral. Escuela de Nutrición y Dietética. Facultad de Salud.

Universidad Santo Tomás. Santiago. Chile.

Av. Ejército $N^{\circ} 146$, Santiago. E-mail: vilmaquitral@santotomas.cl

Este trabajo fue recibido el 19 de marzo de 2019. Aceptado con modificaciones: 24 de septiembre de 2019. Aceptado para ser publicado: 19 de noviembre de 2019.

oven. Chicken, turkey, pork and beef patties were purchased commercially, cooked in a conventional oven and in microwaves. Cooking loss was calculated, rancidity and sensory differences were evaluated between patties of the same type of meat and cooked in the different oven types. Cooking loss was lower in patties cooked in microwaves compared to those cooked in conventional ovens. For both oven types, acidity and peroxide index increased with respect to raw ones. Acidity was higher in hamburgers cooked in conventional ovens and the peroxide index was higher in burgers cooked in microwaves, reaching 10.6 meq $\mathrm{O}_{2} /$ $\mathrm{kg}$. In sensory analysis, significant differences were found $(p<0.05)$ between patties cooked in a conventional oven and in microwaves. It is a better option to cook patties in 
a conventional oven, because the peroxide index is smaller than in microwaves; and within types of patties, beef and pork are more stable against oxidation.

Keywords: Microwave; Patties; Peroxide index; Rancidity; Sensory.

\section{INTRODUCCIÓN}

La energía se puede trasmitir sin desplazamiento de materia, a través de "ondas". Dentro de éstas se encuentran las microondas, con una longitud de pocos centímetros (entre 1 milímetro y un metro). Su frecuencia es de entre $300 \mathrm{MHz}$ y $300 \mathrm{GHz}$ mayor que radiación infrarroja, visible, ultravioleta, rayos X y gamma. Dentro de sus aplicaciones más conocidas se encuentra el horno microondas.

Las microondas son ondas electromagnéticas, que pasan a través del alimento y causan movimiento en las moléculas de agua, con ello se produce fricción y esto provoca el calentamiento ${ }^{1,2}$.

El uso de horno microondas está muy extendido tanto a nivel doméstico, en servicios y a nivel industrial. En este último caso, las microondas se han aplicado en diferentes procesos tecnológicos como deshidratación, pasteurización, esterilización, descongelación, horneo y otras. Sus ventajas son la facilidad y rapidez de operación, baja mantención y manipulación segura ${ }^{3}$. A nivel doméstico se usa para descongelar, calentar y preparar alimentos ${ }^{1}$. Además, se ha reportado que la retención de nutrientes en alimentos calentados en microondas es alta ${ }^{4,5}$, por ejemplo, la retención de tiamina en carne de pollo es mayor al calentar en microondas frente al calentamiento en agua por ebullición ${ }^{2}$.

Sobre el efecto de microondas en la calidad sensorial de alimentos, existen resultados contradictorios, en algunas investigaciones se demuestra que se afecta negativamente $y$ en otras que se conserva ${ }^{3}$; los diferentes resultados pueden atribuirse a que son distintos alimentos, cortes, etc. además de distintos tiempos de exposición.

Respecto a la composición nutricional, Goran y colaboradores $^{6}$ investigaron el contenido de minerales de carne de vacuno y cerdo tratada en horno y en microondas, los investigadores concluyeron que el horno convencional es más efectivo para mejorar los niveles de minerales en la carne.

La concentración de carotenoides parece disminuir al cocinar los alimentos en microondas respecto a otros métodos de cocción, al igual que los glucosinolatos. Mientras que la concentración de polifenoles y vitamina C no se ve afectada ${ }^{7,8}$.

Sobre el efecto de microondas en el sistema antioxidante, un estudio realizado en ratas comprobó que en los animales alimentados con preparaciones en microondas se redujeron significativamente la actividad de superoxidodismutasa y de catalasa, a la vez que disminuyeron las concentraciones de vitamina A y $\mathrm{E}^{9}$.

En el calentamiento en microondas no hay uniformidad en la distribución del campo magnético, por lo tanto, la distribución del calor no es homogénea dentro del alimento y se produce el efecto de sobrecalentamiento del borde de los mismos ${ }^{2,4}$.

Dentro de las desventajas de la aplicación de microondas, es que al calentar alimentos con alto contenido de materia grasa por microondas existe un mayor deterioro frente a métodos tradicionales. Sin embargo, esto va a depender de la temperatura y tiempo utilizado ${ }^{10}$. Algunos investigadores han determinado formación de radicales libres en aceites vegetales, especialmente a altas temperaturas, los que desencadenan la peroxidación e hidrólisis con el consecuente aumento de ácidos grasos libres; disminución de tocoferoles y formación de dímeros y polímeros ${ }^{1,11}$. Se ha detectado incluso aumento del valor de oxidación total (TOTOX) y de viscosidad, además de cambios significativos de color ${ }^{12}$. También se ha demostrado un aumento de la densidad, e índice de refracción en grasas y aceites calentados en microondas. Las microondas también tienen efecto en la distribución de los glóbulos de grasa en los alimentos ya que existe una migración de glóbulos de grasa durante el calentamiento en horno microondas mayor que en calentamiento convencional. Echarte y colaboradores ${ }^{13}$ determinaron que el calentamiento de hamburguesas en microondas provoca mayor oxidación del colesterol que al realizar fritura.

La adopción de hábitos de alimentación poco saludables ha provocado graves problemas en la salud de la población, aumentando la prevalencia de enfermedades crónicas no transmisibles ${ }^{14}$. Dentro de los alimentos poco saludables se encuentran las hamburguesas, que si bien es cierto es un alimento proteico, el contenido de grasa es muy alto. Su consumo ha aumentado en los últimos años, principalmente en personas de 15 a 24 años ${ }^{15}$, y de acuerdo con Araneda y colaboradores $^{16}$, en adolescentes de 14 a 17 años es mayor el consumo en hombres que en mujeres.

La materia grasa de las hamburguesas se altera por efecto del calor resultando en productos de oxidación primarios y secundarios que son tóxicos para el cuerpo humano, ya que son capaces de alterar el metabolismo a nivel celular; además las hamburguesas contienen colesterol, y los productos de oxidación de colesterol (COPs), son altamente tóxicos ${ }^{17}$. Por otra parte, la sal que está presente en alta concentración en las hamburguesas también favorece la oxidación lipídica ${ }^{18}$. De acuerdo con estos antecedentes, es relevante determinar qué tratamiento térmico es más adecuado para atenuar la oxidación. El objetivo del presente trabajo es comparar el efecto de cocción de hamburguesas en microondas y en horno convencional, sobre la pérdida de peso y estabilidad frente a rancidez y oxidación. Además, determinar si existen diferencias significativas desde el punto de vista sensorial.

\section{MATERIAL Y MÉTODOS Muestras}

Se obtuvieron muestras de hamburguesas clásicas de cerdo, pollo, pavo y vacuno (marcas comerciales: Supercerdo, Superpollo, Sopraval y La Preferida respectivamente). Las muestras se obtuvieron congeladas, se trasladaron en una caja 
de material aislante y se almacenaron a $-18^{\circ} \mathrm{C}$ en el laboratorio hasta su análisis. En un supermercado se adquirieron cuatro hamburguesas de cada tipo (correspondientes a un mismo lote), y luego se realizó lo mismo en otros dos supermercados diferentes, obteniendo muestras de hamburguesas de lotes distintos.

\section{Preparación de las muestras}

Las hamburguesas fueron cocidas en horno convencional a gas de marca Fensa en que la transferencia térmica ocurre por convección, por 10 a 15 minutos a una temperatura de $175{ }^{\circ} \mathrm{C}$ y en horno de microondas doméstico marca Thomas (con potencia de 800 Watts), por un tiempo de 5 minutos aproximadamente hasta alcanzar en ambos casos una temperatura de $70^{\circ} \mathrm{C}$ en el centro térmico.

\section{Análisis de Pérdida por cocción}

Se determinó el peso de las hamburguesas crudas (las que fueron previamente descongeladas, manteniéndolas en refrigeración por 24 horas); y cocidas (dejándolas enfriar por 2 horas) y se calculó la pérdida de peso según la fórmula ${ }^{19}$ :

[Peso hamburguesa cruda-Peso hamburguesa cocida] x 100] Pérdida de peso $=$

Peso hamburguesa cruda

\section{Análisis químico: rancidez}

Se extrajo la materia grasa de las hamburguesas (crudas y cocidas) por el método de Bligh y $D_{y e r^{20}}$ en que la muestra finamente molida y homogeneizada se adicionó de cloroformo, metanol y agua destilada. Este es un método de extracción en frío.

En la materia grasa extraída se determinó:

1) Acidez libre: por método volumétrico de acuerdo con $\mathrm{AOCS}^{21}$ y expresado como porcentaje de ácido oleico;

2) Índice de Peróxido: por el método yodométrico ${ }^{22}$ y expresado como miliequivalentes de peróxidos por $\mathrm{kg}$ de aceite.

\section{Análisis sensorial de las muestras}

Se aplicó una prueba para detectar diferencias entre las muestras cocinadas en distinto tipo de horno. Como prueba de discriminación se aplicó la prueba triangular ${ }^{23}$ con 8 evaluadores entrenados. El panel de evaluadores estaba constituido por 6 mujeres y 2 hombres de 21 a 26 años. Los evaluadores del panel sensorial recibieron entrenamiento de acuerdo con la Norma Española UNE-EN ISO $8586^{24}$. Se realizaron 4 sesiones, una sesión para cada tipo de hamburguesa. En cada sesión, el evaluador recibió tres tríos de muestras; en cada trío 2 muestras eran iguales (cocinadas en el mismo horno) y 1 muestra distinta, debiendo identificar la muestra distinta. Las muestras se presentaron recién cocidas con una temperatura interna de $60^{\circ} \mathrm{C}$, como medio de neutralización entre tríos, se entregó una taza de té levemente endulzado. Las muestras se presentaron en platitos codificados con 3 dígitos aleatorios y el orden de las muestras se realizó al azar. Para el análisis de resultados se calcularon las respuestas correctas de todo el panel y se compararon con el valor de la tabla de "mínimo de juicios correctos para establecer diferencias significativas con nivel de significación de 5\%".

\section{Análisis estadístico}

Los datos de porcentaje de pérdida de peso se analizaron estadísticamente con t de Student para muestras no pareadas. Los resultados de acidez e índice de peróxido se analizaron por ANOVA y test de Tukey para establecer diferencias significativas a nivel de 5\%, con el programa SPSS 15.0.

\section{RESULTADOS}

La figura 1 presenta los resultados de pérdida de peso por cocción (\%) de las hamburguesas cocinadas en microondas y en horno convencional. Las pérdidas de peso fueron similares entre ambos tipos de cocción en hamburguesas de cerdo y pavo (15,0\% aproximadamente). En hamburguesas de pollo la pérdida de peso por cocción fue significativamente mayor $(p<0,05)$ cuando se utilizó horno convencional llegando a $25,1 \%$, en comparación a microondas con $16,8 \%$, al igual que en hamburguesas de vacuno, que tuvo pérdida de peso de 16,8 y $11,8 \%$ en horno convencional y en microondas respectivamente.

Los resultados de acidez (Figura 2) indican que ambos calentamientos provocaron aumento de ácidos grasos libres en todos los tipos de hamburguesas respecto a las muestras crudas, siendo significativos $(p<0,05)$ en el caso de las hamburguesas de cerdo, pollo y vacuno cocidas en horno convencional. Se observa además que la acidez tendió a ser mayor en las hamburguesas cocidas en horno convencional. Las hamburguesas de pollo y cerdo fueron las que presentaron mayor acidez, tanto en cocción en microondas como en horno convencional.

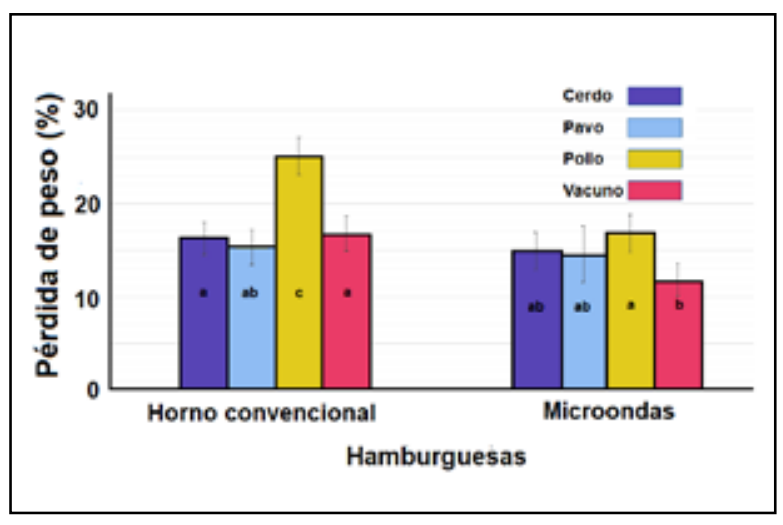

Figura 1: Pérdida por cocción de hamburguesas cocidas en horno convencional y en microondas. Letras distintas indican diferencias significativas entre las muestras $(p<0,05)$. 
En la figura 3 se presenta la variación del índice de peróxido en las muestras de hamburguesas, cocinadas en ambos tipos de horno respecto a las muestras crudas. En microondas los valores de índice de peróxido fueron significativamente mayores $(p<0,05)$ para las hamburguesas de pavo y pollo, respecto a los valores alcanzados en horno convencional; en el caso de las hamburguesas de cerdo y vacuno propendieron a aumentar en microondas respecto a horno convencional. Las hamburguesas de pollo presentaron alto índice de peróxido en crudo, y en cocido aumentan aún más.

Los resultados de análisis sensorial se presentan en la tabla 1, e indican que existieron diferencias

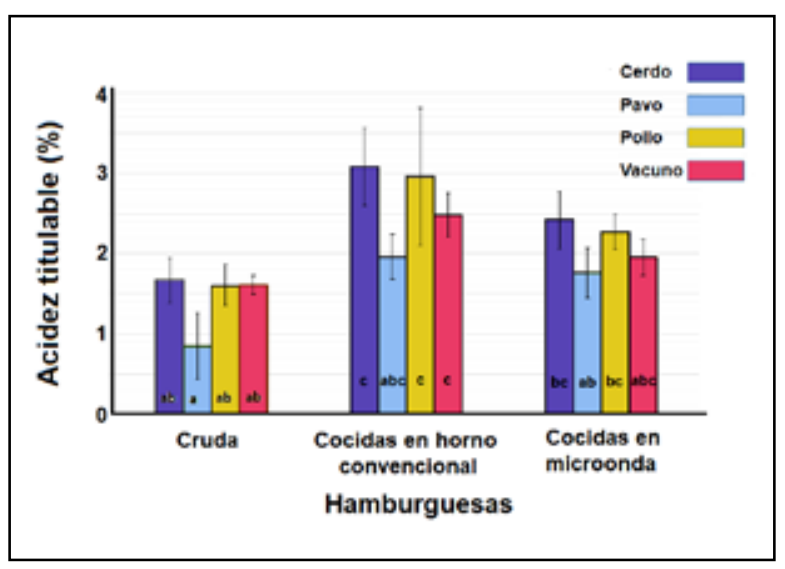

Figura 2: Acidez de materia grasa de hamburguesas cocidas en horno convencional y en microondas. Letras distintas indican diferencias significativas entre las muestras $(p<0,05)$. significativas entre hamburguesas cocidas en microondas y en horno convencional, ya que para todos los tipos de hamburguesas (pollo, pavo, cerdo, vacuno), los aciertos de los evaluadores fueron mayores que el número mínimo de juicios correctos para establecer diferencias significativas $(p<0,05)$ en la tabla de significancia para prueba triangular.

\section{DISCUSIÓN}

Los valores de pérdida por cocción fueron inferiores a los que se han reportado en otros estudios, como en hamburguesas de cerdo cocidas en horno con aire caliente que tuvieron 20,4\% de pérdida ${ }^{25}$. En hamburguesas de vacuno

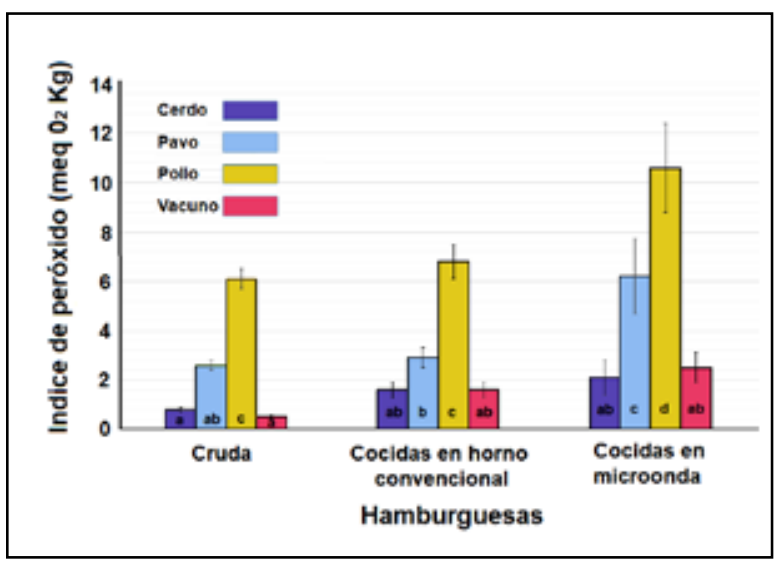

Figura 3: Índice de peróxido de materia grasa de hamburguesas cocidas en horno convencional y en microondas. Letras distintas indican diferencias significativas entre las muestras $(p<0,05)$.

Tabla 1. Resultados de prueba triangular.

\begin{tabular}{|lccc|}
\hline Tipo de hamburguesa & Juicios correctos & $\begin{array}{c}\text { Mínimo juicios correctos } \\
\text { para establecer diferencias (5\%) }\end{array}$ & p \\
\hline Cerdo & 14,0 & 13,0 & $<0,05$ \\
Pavo & 16,0 & 13,0 & $<0,05$ \\
Pollo & 20,0 & 13,0 & $<0,05$ \\
Vacuno & 17,0 & 13,0 & $<0,05$ \\
\end{tabular}


se han reportado mayores pérdidas por cocción en hornos de convección, llegando a 40,326 y 34,6\% ${ }^{27}$.

Por el contrario, existen otros estudios que encontraron mayor pérdida por cocción en microondas en comparación con otros métodos de preparación ${ }^{19,28}$. Domínguez y colaboradores $^{28}$ sugieren que se debe que al calentar en microondas no se forma una cubierta en la superficie del alimento que evita la pérdida de humedad, lo que si se produce al calentar en horno convencional.

La pérdida de peso por cocción se debe a evaporación de agua por la temperatura, y a la liberación de agua posterior al proceso de calentamiento, en que el calor induce desnaturalización proteica, provocando desligamiento del agua que se encuentra dentro de las estructuras proteicas ${ }^{29}$. Los valores encontrados en calentamiento por microondas en este estudio son similares a los determinados por Echarte y colaboradores ${ }^{13}$ en hamburguesas de pollo y vacuno; y mucho menores que los determinados en carne de pollo y cordero por Kumar y Aalbersberg 5 .

La mayor acidez de las hamburguesas calentadas en horno convencional se puede atribuir a que en ese tipo de horno el calentamiento es más lento respecto a microondas, lo que puede favorecer la reacción de hidrólisis porque ésta requiere la presencia de agua y de enzimas lipolíticas. El tratamiento térmico provoca modificación en los lípidos, el mayor efecto se produce en los ácidos grasos poliinsaturados de cadena larga (PUFA) del tejido adiposo mediante la oxidación de lípidos².

El calor específico de grasas y aceites es bajo, lo que provoca que se calienten rápidamente, y por este motivo son tan sensibles a los tratamientos térmicos. Al calentar aceites vegetales en microondas se produce mayor alteración oxidativa de los lípidos que al calentar en horno convencional e incluso en fritura. Esto se traduce en aumento del índice de peróxido en los primeros tiempos de calentamiento, para luego descender y aumentan los productos de oxidación secundaria como el índice de p-anisidina y sustancias reactivas al ácido tiobarbitúrico (TBARs). También es mayor la formación de radicales libres, ácidos grasos libres por hidrólisis, formación de dímeros y polímeros, disminución de tocoferoles e isomerización de ácidos grasos a la forma $\operatorname{trans}^{1,11,30,31}$. Aydinkaptan y Barutçu Mazi ${ }^{32}$ demostraron que al freir en microondas los valores de viscosidad e índice de refracción son mayores, los compuestos polares y ácidos grasos libres aumentaron significativamente respecto a la fritura en profundidad.

Las hamburguesas de pavo y pollo contienen mayor proporción de ácidos grasos poliinsaturados que cerdo y vacuno, lo que justificaría el mayor índice de peróxido en esas hamburguesas.

Los valores de índice de peróxido concuerdan con otros autores que determinaron compuestos primarios y secundarios de oxidación. Badiani y colaboradores ${ }^{33}$ trataron térmicamente cortes de res y encontraron que el valor peróxido no tuvo diferencias significativas entre horno convencional y horno microondas. Mientras que TBARs, que corresponde a un compuesto secundario de oxidación, fue mayor en la carne preparada en microondas. Por otra parte, el índice de TBARs aumentó en la carne de cerdo ibérico cocida, alcanzando el valor más alto en microondas (1,35 mg MDA/kg músculo), el mismo efecto se produjo en carne de potro, ya que TBARs fue mayor al calentar en microondas, respecto a horno convencional ${ }^{28}$ al igual que en carne de pollo ${ }^{34}$. Los compuestos secundarios de oxidación se han relacionado con aterosclerosis, enfermedad de Alzheimer, inflamación y procesos de envejecimiento ${ }^{35}$. Al comparar el contenido de productos de oxidación del colesterol en hamburguesas de pollo y res cocinadas en microondas y fritas en aceite de oliva, se demostró que en microondas se produce un número significativamente mayor de estos compuestos comparados con muestras crudas y muestras fritas ${ }^{13}$. Incluso la oxidación de proteínas es mayor en microondas como lo demostraron Soladoye y colaboradores $^{36}$ en preparación de tocino.

Desde el punto de vista nutricional, es muy importante considerar el tratamiento de cocción que se aplica a los alimentos, ya que, en algunos casos, disminuye la concentración de nutrientes, o su biodisponibilidad; y en este caso se produce rancidez, con generación de compuestos nocivos para la salud.

En cuanto al análisis sensorial, se encontraron diferencias entre muestras de hamburguesas calentadas en microondas y en horno. Jouquand y colaboradores ${ }^{37}$ prepararon un guiso de carne de vacuno, al comparar la calidad sensorial de la preparación en microondas respecto a la preparación tradicional (en horno de convección), detectaron que en apariencia y sabor no existieron diferencias significativas, sin embargo, la textura de la carne fue significativamente superior en la preparación tradicional. En el caso de la preparación en microondas, la carne se calificó de "poco masticable" y "muy seca".

En cuanto a la disminución del sabor y aroma en carnes y productos cárnicos preparados en microondas, se asocia a la ausencia de reacciones de pardeamiento no enzimático, ya que al ser un calentamiento tan rápido muchos compuestos volátiles quedan retenidos en la matriz alimentaria sin poder reaccionar, lo que en horno convencional si ocurre debido al calentamiento más lento. En microondas el calentamiento no es uniforme, no se desarrollan colores, aromas, sabores y se afecta la textura de los alimentos ${ }^{38}$. Respecto a la textura, Rababah y colaboradores ${ }^{34}$ evaluaron por métodos instrumentales la fuerza de corte, fuerza de cizallamiento, dureza, elasticidad y cohesividad en carne pollo cocinada en microondas y en horno convencional; los autores demostraron que existieron diferencias significativas $(p<0,05)$ en los valores, lo que indica mejor calidad de textura de la carne preparada en horno convencional.

\section{CONCLUSIÓN}

De acuerdo con los resultados, se demuestra que la mayor pérdida por cocción ocurre en hamburguesas de pollo. Las hamburguesas calentadas en horno sufren más 
hidrólisis lipídica y las calentadas en microondas sufren mayor oxidación lipídica. La hamburguesa de pavo sufre menor hidrólisis lipídica, mientras que la de vacuno y cerdo menor oxidación. Existen diferencias significativas $(p<0,05)$ en la calidad sensorial entre hamburguesas calentadas en horno convencional y en microondas, siendo mayormente afectada la textura.

Resulta mejor opción cocinar las hamburguesas en horno convencional, ya que es menor la oxidación de la materia grasa, además las hamburguesas de vacuno y cerdo resultaron ser más estables frente a esta alteración.

Agradecimientos. A la Srta. Oriana Salazar Álvarez por su colaboración en la realización del estudio.

\section{BIBLIOGRAFÍA}

1. Chiavaro E, Rodriguez-Estrada MT, Vittadini E, Pellegrini N. Microwave heating of different vegetable oils: Relation between chemical and thermal parameters. LWT - Food Sci Technol. 2010; 43(7): 1104-1112.

2. Barbosa-Cánovas G, Medina-Meza I, Candoğan K, BermúdezAguirre D. Advanced retorting, microwave assisted thermal sterilization (MATS), and pressure assisted thermal sterilization (PATS) to process meat products. Meat Sci. 2014; 98(3): 420-434.

3. Chandrasekaran S, Ramanathan S, Tanmay Basak. Microwave food processing - A review. Food Res Int. 2013; 52 (1): 243-261.

4. Chizoba Ekezie F-G, Sun D-W, Han Z, Cheng J-H. Microwaveassisted food processing technologies for enhancing product quality and process efficiency: A review of recent developments. Trends Food Sci Tech. 2017; 67: 58-69.

5. Kumar S, Aalbersberg B. Nutrient retention in foods after earth-oven cooking compared to other forms of domestic cooking. Proximates, carbohydrates and dietary fibre. I Food Compos Anal. 2006; 19(4): 302-310.

6. Goran GV, Tudoreanu L, Rotaru E, Crivineanu V. Comparative study of mineral composition of beef steak and pork chops depending on the thermal preparation method. Meat Sci. 2016; 118: 117-121.

7. Bureau S, Mouhoubi S, Touloumet L, Garcia C, Renard C. Are folates, carotenoids and vitamin $C$ affected by cooking? Four domestic procedures are compared on a large diversity of frozen vegetables. LWT-Food Sci Technol. 2015; 64(2): 735-741.

8. Tian J, Chen J, Lv F, Chen S, Ye X. Domestic cooking methods affect the phytochemical composition and antioxidant activity of purple-fleshed potatoes. Food Chem. 2016; 197(Pt B): 1264-1270.

9. Eke B, Jibiri N, Bede EN, Anusionwu B, Alisi C. Effect of ingestion of microwaved foods on serum of anti-oxidant enzymes and vitamins of albino rats. I Radiat Res. 2017; 10(2): 148-151.

10. Malheiro R, Oliveira I, Vilas-Boas M, Falcão S, Bento A, Pereira JA. Effect of microwave heating with different exposure times on physical and chemical parameters of olive oil. Food Chem Toxicol. 2009; 47: 92-97.

11. Hassanein MM, El-Shami SM, El-Mallah MH. Changes occurring in vegetable oils composition due to microwave heating. Grasas Aceites. 2003; 54(4): 343-349.

12. Sobhani A, Mohammed AS, Ghobakhlou F, Ghazali HM.
Determining the oxidative stability and quality of tiger nut (Cyperus esculentus) oil and its antioxidant activity during microwave heating. Rev Esp Nutr Hum Diet. 2018; 22(1): 52-63.

13. Echarte M, Ansorena D, Astiasarán I. Consequences of microwave heating and frying on the lipid fraction of chicken and beef patties. J Agric Food Chem. 2003; 51 (29): 5941-5945.

14. Ponce C, Pezzotto E, Compagnucci AB. Eating habits of medical students of the National University of Rosario, Argentina. Rev Chil Nutr. 2019; 46(5): 554-560.

15. Zapata L. Aguilera N. Hamburguesas - Composición nutricional de hamburguesas de vacuno, pollo y cerdo. Editorial ODECU. Santiago, Chile. 2018.

16. Araneda J, González D, Mella V, Pérez K, Quezada G, Pinheiro AC. High-protein foods intake by adolescents in Chillan, Chile. Rev Chil Nutr. 2019; 46(3): 295-302.

17. Maldonado-Pereira L, Schweiss M, Barnaba C, MedinaBaeza IG. The role of cholesterol oxidation products in food toxicity. Food Chem Toxicol. 2018; 118: 908-939.

18. Mariutti L, Bragagnolo N. Influence of salt on lipid oxidation in meat and seafood products: A review. Food Res Int. 2017; 94: 90-100.

19. Lorenzo JM, Cittadini A, Munekata PE, Dominguez R. Physicochemical properties of foal meat as affected by cooking methods. Meat Sci. 2015; 108: 50-54.

20. Bligh E, Dyer W. A rapid method of total lipid extraction and purification. Can J Biochem Physiol. 1959; 37(8): 911917.

21. American Oil Chemists's Society A.O.C.S. Official methods and recommended practices of the American oil chemists' society. Champaign, IL: AOCS Press. 1993.

22. UNE-EN ISO 3960:2010. Animal and vegetable fats and oils - Determination of peroxide value - Iodometric (visual) end point determination (ISO 3960:2007, corrected version 2009-05-15).

23. UNE-EN ISO 4120:2008. Sensory analysis - Methodology - Triangle test (ISO 4120:2004)

24. UNE-EN ISO 8586:2014. Sensory analysis - General guidelines for the selection, training and monitoring of selected assessors and expert sensory assessors (ISO 8586:2012).

25. Danowska-Oziewicz M, Kurp L. Physicochemical properties, lipid oxidation and sensory attributes of pork patties with lupin protein concentrate stored in vacuum, modified atmosphere and frozen state. Meat Sci. 2017; 131: 158-165.

26. Cox S, Abu-Ghannam N. Enhancement of the phytochemical and fibre content of beef patties with Himanthalia elongata seaweed. Int J Food Sci Technol. 2013; 48(11): 2239-2249.

27. López-López I, Cofrades S, Cañeque V, Díaz MT, López O, Jiménez-Colmenero F. Effect of cooking on the chemical composition of low-salt, low-fat Wakame/olive oil added beef patties with special reference to fatty acid content. Meat Sci. 2011; 89 (1): 27-34.

28. Domínguez R, Gómez M, Fonseca S, Lorenzo JM. Effect of different cooking methods on lipid oxidation and formation of volatile compounds in foal meat. Meat Sci. 2014; 97(2): 223-230.

29. Juárez M, Failla S, Ficco A, Peña F, Polvillo O. Buffalo meat composition as affected by different cooking methods. Meat Sci. 2010; 88(2-3): 145-148.

30. Bagher Hashemi SM, Mousavi Khaneghah A, Koubaa M, Lopez-Cervantes J, Asadifard S. Novel edible oil sources: Microwave heating and chemical properties. Food Res Int. 
2017; 92: 147-153.

31. Rękas A, Siger A, Wroniak M. The effect of microwave pre-treatment of rapeseed on the degradation kinetics of lipophilic bioactive compounds of the oil during storage. Grasas Aceites. 2018; 69(1): e233.

32. Aydinkaptan E, Barutçu Mazı I. Monitoring the physicochemical features of sunflower oil and French fries during repeated microwave frying and deep-fat frying. Grasas Aceites. 2017; 68(3): e233.

33. Badiani A, Stipa S, Bitossi F, Gatta PP, Chizzolini R. Lipid composition, retention and oxidation in fresh and completely trimmed beef muscles as affected by common culinary practices. Meat Sci. 2002; 60(2): 169-186.

34. Rababah TM, Ereifej KI, Al-Mahasneh MA, Al-Rababah MA. Effect of plant extracts on physicochemical properties of chicken breast meat cooked using conventional electric oven or microwave. Poultry Sci. 2006; 85(1): 148-154.

35. Rasinska E, Rutkowska J, Czarniecka-Skubina E, Tambor $K$. Effects of cooking methods on changes in fatty acids contents, lipid oxidation and volatile compounds of rabbit meat. LWT - Food Sci Technol. 2019; 110: 64-70.

36. Soladoye OP, Prieto N, Lopez-Campos O, Aalhus JL, Juárez $M$. Potential of near infrared (NIR) spectroscopy and dual energy X-ray absorptiometry (DXA) in predicting pork belly softness. Meat Sci. 2018; 142: 1-4.

37. Jouquand C, Tessier F, Bernard J, Marier D, Laguerre JC. Optimization of microwave cooking of beef burgundy in terms of nutritional and organoleptic properties. LWT - Food Sci Technol. 2015; 60(1): 271-276.

38. Ozkoc SO, Sumnu G, Sahin S. Recent Developments in Microwave Heating. Emerging Technologies for Food Processing (Second Edition). 2014; pp. 361-383. 